\title{
A Novel Framework for Speech Signal Denoising using PSO Optimized ICA-DWT Algorithm
}

\author{
S. Lakshmikanth' ${ }^{1}$, K. R. Natraj ${ }^{2}$ and K. R. Rekha ${ }^{2}$ \\ 'Department of Electronics and Communication Engineering, Vivekananda Institute of Technology, Kumbalagodu, \\ Kengeri, Bengaluru - 560074, Karnataka, India; lakshmikanth18@gmail.com \\ 2Department of Electronics and Communication Engineering, SJB Institute of Technology, No.67, BGS Health and \\ Education City, Dr. Vishnuvardhan Road, Kengeri, Bengaluru 560060, Karnataka, India; \\ natraj.sjbit@gmail.com, rekha.sjbit@gmail.com
}

\begin{abstract}
Objectives: In this paper a hybrid approach of ICA-DWT algorithm optimized using Particle Swarm Optimization (PSO) is proposed to deal with problems of aperiodic and period noises in industrial noise environment. Method/analysis: The feature of Independent Component Analysis (ICA) for separating the signals of various channels is exploited to separate noise peaks from the speech channel. To reserve the original signal and discern the noise, the speech is segmented in various levels of frequencies via discrete wavelet transform. The adaptive filtration through wavelet filters has been a powerful tool for signal segmentation into various frequencies. The output of ICA is sourced to Discrete Wavelet Transform (DWT) and is optimized using PSO to generate threshold value and number of wavelets for it. Findings: The results indicate that additional overhead computation of DWT has a better Signal-to-Noise Ratio (SNR) value compared to clean fast ICA algorithm and thus validate the improvement in speech signal intelligibility and quality.For the range of input signals and noise environment, the optimization of PSO to filter the speech signals has best SNR compared to conventional algorithm. Novelty/ Improvement: In the proposed denoising model two stages optimized filtering is presented. The number of wavelet levels and value of threshold is depicted using objective function to minimize Spectral Noise Density (SND). The objective function is optimized with PSO in constraints of SND to generate the best possible levels of DWT and thus maximize the SNR ultimately.
\end{abstract}

Keywords: DWT, ICA, PSO, Speech Signals

\section{Introduction}

The speech signals lies in the category of acoustic signals represented in digital mode generally studied in digital signal processing. The undesired signals from various sources combine with speech signals and result in noise. The reasons for noise in digital communication are finite precision of equipment or use of local components, faults in lines and deficiency in coding etc. The study of such type of noise is a different subject as they alter the nature of speech signal. The acoustic noise always affects the speech signal in free space since surrounding noise is always present. These noises are the audible signals that result due to any operation, human or by equipment. The test parameters $\mathrm{SQI}^{1}$ and $\mathrm{SNR}^{2,3,4}$ are the direct measure of noise content present in speech signals. $\operatorname{In}^{1}$ first attempted for signal denoising by suppressing the noise from the source signal. However for speech signal filtering, both noise and signal are assumed to be stationary and their static behavioural is estimated prior. The above assumed conditions are impossible to withstand in current era of communication. In last few decades the wireless channels

${ }^{*}$ Author for correspondence 
have become noisier in nature. Due to above said issues the research related to acoustic noise have seen a steep rise.

The industrial noise is generally the machine noise originated in their operation mode. The noise is classified in periodic and aperiodic noise. Periodic noise has explicit characteristics to repeat its behaviour after every fixed interval of time. For instance in internal combustion engine noise: the engine produces a particular noise for one cycle of revolution (equation 1(a)). In the other cycle the sound from engine is similar of first cycle for same time period, frequency and amplitude (equation 1(b)).

$$
\begin{gathered}
y_{1}=x(t) \\
y_{2}=y_{1}
\end{gathered}
$$

Therefore engine sound for the time interval $t$ is given as the summation of all the sound instances of same property where, $y$ is the periodic noise.

$$
\sum y=y_{1}+y_{2} \cdots y_{n}=x(t)
$$

Aperiodic noise is the random signals with high intensity. For example noise of a machine: the noise of machine originates due to its operation on a subject. The design and the properties of a subject can be different such as pressure applied on it. The nature of sound in such case cannot be predicted and does not replicate itself over a period of time. In terms of many applications (security, authentication etc.) the speech signal filtering has been a fundamental subject of research and is well documented in many literatures.

The literature on the ICA and rippling ways exists primarily within the setting of aperiodic noise. The ICA methodology solely generates the freelance elements and therefore needs an extra filter to denoise the signal. Speech improvement with missing TF was supported $\mathrm{ICA}^{5}$ as ICA will be data-driven, adjustive and linear illustration in nature. The mix of ICA with wiener filter ${ }^{6}$ was wont to minimize mean sq. error, strained ICA with Bessel options ${ }^{7}$ to extract subsets of desired freelance supply signals from a group of mixture of supply signals. ICA with adaptive filters ${ }^{\underline{8}}$ was used for speech improvement exploiting the properties of ICA and filtering the elements before reconstruction. In tested Bayesian algorithmic rule ${ }^{9}$ with ICA for Max A Posteriori (MAP) computer and transformation of learned speech knowledge via ICA. The restrictions of linear filters are already mentioned and performance of adjustive filters like rippling filters depends on its application. Consistent with the soft thresholding techniques ${ }^{10}$ perform higher than onerous ways. Specific rippling techniques like Haar associate degreed Daubechies in an experimentation showed mixed response for signals in vary 8-20 sound units. Adjustive wavelets $\underline{12}$, strong rippling denoising ways ${ }^{13}$ is some improvement techniques experimented in same reference. In Bayesian denoising methodology ${ }^{11,12}$, best signal calculable for generation of minimum mean sq. estimators for specific options of speech signal. Additionally with wavelet ${ }^{\frac{13}{3}}$ assumes a marginal native regularity interprets into constraints on the multifractal spectrum of the signal. The theoretical approach for this can be supported Holder exponent during a random frame.

The organization of paper is as follows: the proposed algorithm discusses the mathematical model of architecture and the following sections describe the tools used in this paper along with subsequent theory. The experimental setup discusses the simulation parameters and nature of input signals followed by discussion of noise, sourced for experimentation. The results prove the efficiency of this work comparing proposed algorithm with ICA and ICA-DWT methods and a conclusion is supported at the end of this paper.

\section{Proposed Denoising Model}

The natural phenomenon of speech signals mixing with noise is replicated in a virtual environment of Matrix Laboratory (MATLAB). The speech signal is mixed with noise signal and a new signal $(z(t)$ $z(t))$ is generated. This signal is the input for testing algorithms and filtering mechanism proposed in this paper. The solitary property of ICA to un-mix two signals (originated from separate sources) is exploited to generate a waveform of speech signals. However, preliminary experiments on speech signals obtained at output of ICA detected presence of noise amplitudes in its waveform. To reserve the original signal and discern the noise, the speech is segmented in various levels of frequencies via discrete wavelet transform. In initial experiment three level wavelets are selected as shown in Figure 2 and soft thresholding filtered the signals. The SNR of these signals are recorded and compared with outputs of second experiment where 
the number of wavelet levels and value of threshold is depicted using objective function to minimize SND. The objective function is optimized with PSO in constraints of SND to generate the best possible levels of DWT and thus maximize the SNR ultimately. The SNR of ICA, ICA-DWT and ICA-DWT-PSO are compared to allocate the worthiness of each method in the race of superior technique for denoising. Figure 1 represents the general flow of proposed technique omitted with mathematical details and number of repeated experiments. The DWT filtering in initial filtering possesses an add-on of soft thresholding and PSO in second experimentation defines the levels and threshold coefficients of DWT.

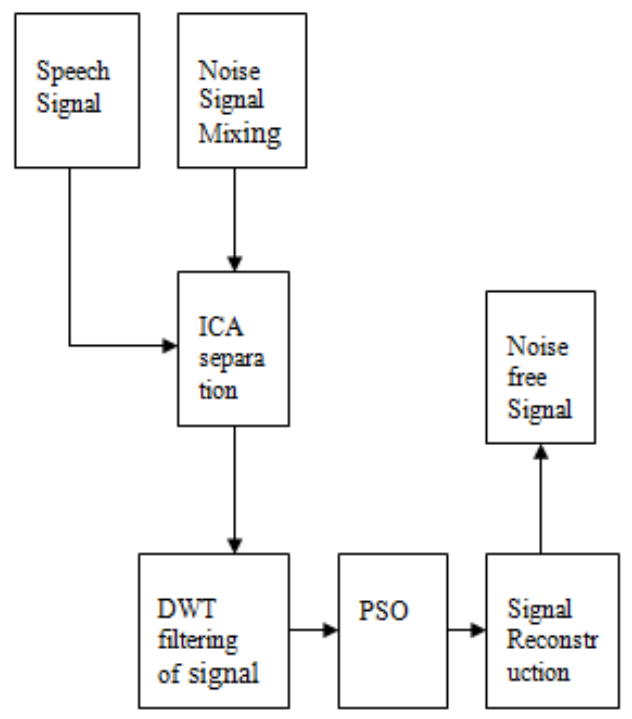

Figure 1. Flow diagram of proposed denoising hybrid model.

\subsection{Independent Component Analysis}

ICA is a blind source separation method that tends to separate mutually independent source signals by exploiting linear mixtures and in absence of mixing coefficients. The input signal of ICA is $z(t)$, which is nothing but the sum of considered speech signal $(x(t))$ and noise $(y(t))$ of environment.

$$
z(t)=x(t)+y(t) z(t)=x(t)+y(t)
$$

The input signal $z(t)$ is a mixed speech and noise signal and intensity and proportion of noise in this signal are random factors. To suite this context, the blind source separation technique seems feasible as the mixing process of signals cannot be elaborated. Considers the input signal $z(t)$ as the original signal $x(t)$ multiplied by a mixing matrix A.

$$
Z=A X
$$

Here, $\mathrm{Z}$ and $\mathrm{X}$ are the matrix representation of input signal and speech signals respectively. ICA tends to determine the speech signal from input signal via inversing the mixing coefficient $\mathrm{W}\left(W=A^{-1}\right.$ ) (equation 5).

$$
S=W Z \cong X
$$

The output $\mathrm{S}$ instead of $\mathrm{X}$ denotes the best possible approximation of $\mathrm{X}$ as the $100 \%$ efficiency of any system is a theoretical hypothesis. The operation of ICA assumes 5 hypotheses, well described in15. Fast ICA and Infomax ICA are two algorithms most popular to measure the independence of components, but considering the fact that signals for experimentation is a single speech and noise signal, the algorithms and their measures are omitted (out of scope in this paper). The signal $S$ or $s(t)$ being statically independent with that of noise determines the square mixing matrix A. The output of ICA is thus an independent speech signal with proportions of noise in it.

\subsection{Discrete Wavelet Transform}

The denoising of speech signal is dependent on the fact that noise artifact are present in form of abrupt peaks in original signal. As the name indicates the signal is sampled into wavelets in discreet manner and has key advantage of temporal resolution over Fourier transform. The Daubechies wavelet method based on the use of recurrence relations samples the mother wavelet into finer samples with each sample having resolution double that of mother wavelet. The components of a wavelet are divided precisely half of previous component and are arranged in upper half (Detailed Coefficients) and lower half (Approximate Coefficients) as shown in Figure 2.

$$
\begin{aligned}
& S_{i}=\frac{1}{\sqrt{M}} \sum_{n} s(t) \varphi_{i}(n) \\
& S_{j}=\frac{1}{\sqrt{M}} \sum_{n} s(t) \emptyset(n)
\end{aligned}
$$




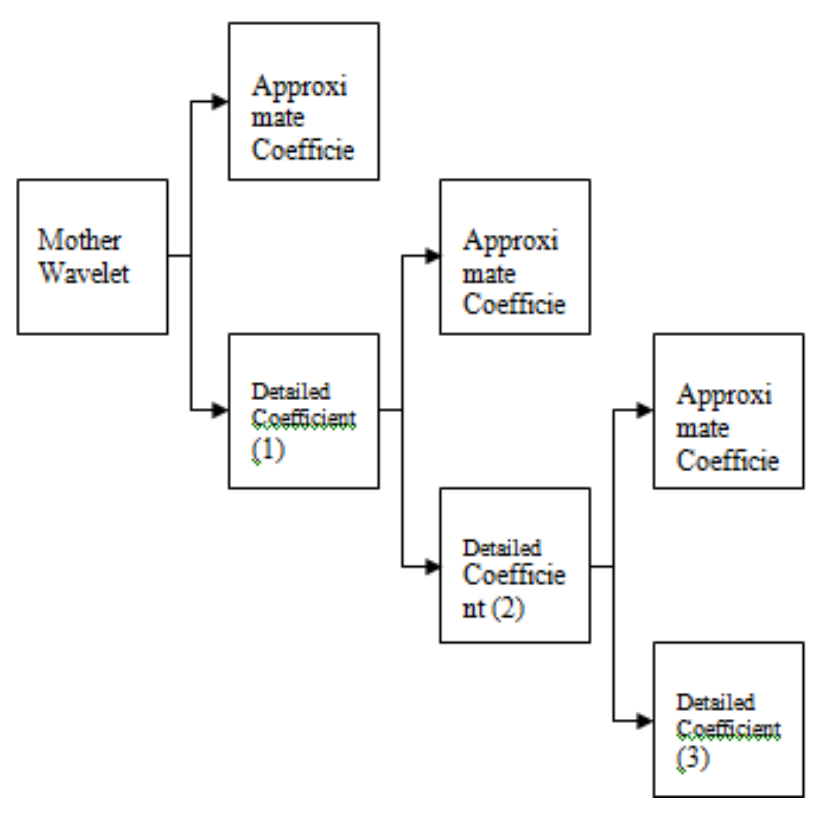

Figure 2. 3-Level discreet wavelet transform for speech signal cascading into various frequency components.

Here, $i \geq j, s(t)$ is the output of ICA and $\mathbf{\mathbf { o }}_{i}(n), s(t) \propto(n)$ are the functions of discrete variables $n=0,1, \ldots M-1$. Equation 6 represents the approximate coefficients and Equation 7 represents detailed coefficients.

The Approximate Coefficients of wavelet is more scaled in second level AC and DC as shown in Figure 2. The removal of noise in DWT is performed by soft thresholding techniques considering its advantages over hard one. In this method the denoising of a Detailed Coefficients is given by16:

$$
S_{j}=\dot{o} \sqrt{2 \log N}
$$

The ${ }^{i^{\text {th }}}$ level of Detailed Coefficients is an orthonormal function against the noise function having variance $\sigma . \mathrm{N}$ is the values of frequency component in Detailed Coefficients and this estimation.

\subsection{Objective Function}

A particle of probable value of $\mathrm{T}$ (threshold) and $\mathrm{L}$ (number of levels in decomposition) for PSO are positive real values. After the soft thresholding and inverse DWT a signal $W W$ is obtained that has greater SNR compared to that of original signal. Hence objective function is given by

$$
\begin{aligned}
& \arg M a x \Rightarrow S N R=\int(T, L) \circ f y(t) \\
& W=D W T(y(t)) \text { of level } L \\
& W=\left\{\begin{array}{cr}
\operatorname{sqn}(W)(|W-T|) \text { for } \hat{y}(t) \geq T \\
\mathbf{0} \quad \text { for } \hat{y}(t)<0
\end{array}\right.
\end{aligned}
$$

The SNR of function $W$ is given by

$$
\left.S N R=\left.\left(\sum \mid W\right)\right|^{\uparrow} 2\right) /\left(\sum|W|^{\uparrow} 2 \quad \sum|y(t)|^{\uparrow^{2}}\right)
$$

Hence, the values of $\mathrm{L}$ and $\mathrm{T}$ through optimization will be selected to achieve maximum SNR.

\section{Particle Swarm Optimization}

PSO is a heuristic approach originally proposed by $\frac{17,19}{2}$. This iterative process as shown in Figure 3 evaluates the candidate solution of current search space. The candidate solution lies in the fitness landscape and determines minimum and maximum of objective function and hence from equation 11 to satisfy equation 12 , the fitness function is:

$$
\text { Maximize } f(k)=W-\widehat{W}
$$

This function can be solved using PSO with constraints of voice quality. The PSO generates the random value of $\mathrm{k}$ as the initial solution. Using fitness function PSO can generate $f(k)$ that is equal to value of $k$. These candidates are referred because the best value for its position and best solution up to now for given drawback in the cluster. PSO maintains the most effective fitness value achieved among all particle in swarm as the individual best fitness. The individual best fitness value of every individual $(f(k))$ is compared and global fitness value is generated. As the information about objective function is not acceptable in inputs of PSO algorithm, hence the distance of solution from local and global maximum and minimum is random and not known to user19. The values of gbest are sourced to the equation of velocity (equation 11) and position (equation 12) and the candidate solution maintains their position and velocity and the fitness value is updated at every stage of iteration:

$$
v_{i}(t+1)=W v_{i}(t)+c_{1} r_{1}\left[\hat{x}_{i}(t)-x_{i}(t)\right]+c_{\mathbf{2}} r_{2}\left[g(t)-x_{i}(t)\right]
$$

Here, $v_{i}(t+1)$ is the velocity of ${ }^{i \text { th }}$ particle at $t+1$ iteration, $c_{\mathbf{1}}$ and $c_{\mathbf{2}}$ are acceleration coefficients, $r_{\mathbf{1}} r_{\mathbf{1}}$ and 
$r_{2}$ are the random number between 0 and 1 . The position of particle is calculated as 12 :

$$
p_{i}(g+1)=p_{i}(g)+v_{i}(g+1)
$$

The solutions in the system are accelerated to new positions by adding new velocities to their positions. The update of velocity and position of the particle till stopping condition is met is responsible for the optimization ability of the PSO algorithm. This process is iterated for 100 times and the value of $g$ best at $100^{\text {th }}$ iteration is the output of PSO.

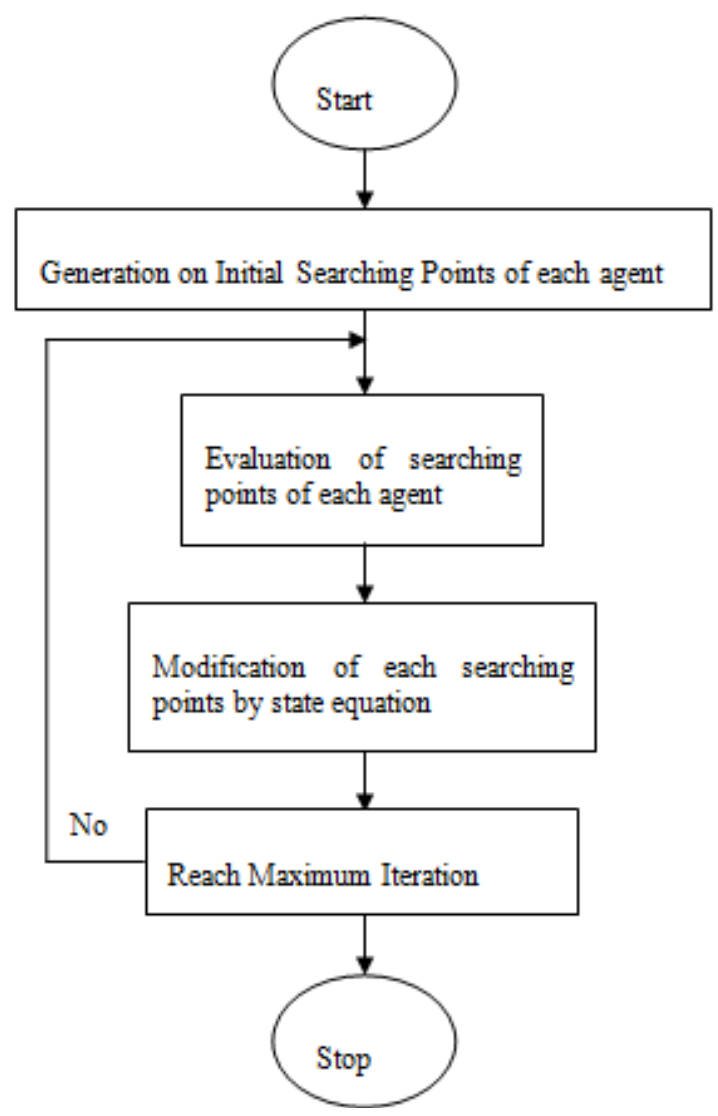

Figure 3. Flow process of PSO.

The output of DWT is inversed to generate the speech signal filtered with noise (equation 16). The comparison of SNR for regenerated speech signal and original speech signal deciphers the effectiveness of proposed method.

$$
s(n)=\sum_{n} s_{\overline{0}}(i) \overline{\mathbf{o}}_{i}(n)+\sum_{j=0}^{\infty} \sum_{n} s_{\hat{0}}(j) \boldsymbol{\sigma}_{j}(n)
$$

\section{Experimental Setup}

Out of the many, we've got chosen 3 parameters to acknowledge the potency of projected work. These parameters area unit chosen considering the actual fact that they're on the market in most of parallel researches and so are normal tool if anyone needs to check the novelty of projected work with existing methods $\frac{18,19}{}$.

\subsection{Signal Quality Index}

Signal quality index is the spectral flatness of a signal given by standard difference in following equation.

$$
W=\log \left\{e_{e} \frac{d f \log (s(f))}{\int d f s(f)}\right\}
$$

\subsection{Signal to Noise Ratio}

The SNR is a measure of low magnitude waves stimulated by some sinusoid approach. The input wave in space-time domain is studied via periodogram optimized with Kaiser Window to attenuate large side lobes. The algorithm looks for the non-zero spectral component to result fundamental frequency. The central moment of each subject is computed for all adjacent bins in a decreasing order (i.e. from maximum to minimum). These frequencies are detectable in second bin and further frequencies are the replica of these steps. The power of a signal is selected as the larger harmonic in case if the signal shows the monotonically decreasing behavior compared with neighbouring signal. The function is the ratio of noise intensity in noise contaminated region derived via median power. To calculate the performance, DC component is rejected and noise at every step could be either ordinate of a point or estimated level. This noise is eliminated for an artifact free signal ${ }^{20}$.

\subsection{Test Inputs}

\subsubsection{Periodic Noise}

Periodic noise is continuous noise with repetitive waveforms of a particular length. The epochs of those waveforms are the disturbances created attributable to repetitive activity of a machine ${ }^{19}$. The frequency of this wave is that variety of epochs generated per unit time. The noise is usually expressed in 'Hertz' and if the noise is audible, then it lies within 20-20,000 Hz. Testing in terms of speech signal following noise and speech signals are thought of for experimentation- 
Noise: a) Diesel Engine b) Machinery c) Factory d) Machinery

Speech Signals: a) Welcome b) Hello Girl c) Lalala

\subsubsection{Non-periodic Noise}

Every single noise that doesn't replicate itself in same fashion and same interval of time is assessed as non-periodic noise. The character of this noise is random and also the wave shape persist high unsymmetrical amplitudes. The supply of those signals might be something together with the mixture of three or additional periodic noises.

\section{Simulation Results}

The results of periodic noise are subsequently arranged for diesel engine, car, machinery and factory noise respectively. The tables below after figures are comparative analysis for three speech signals in each noise domain. The input parameters of each test are same hence the explanation is limited only to Figure 4(a, b, c, d). Similarly the tables against them will indicate the respective SNR and SQI for each speech signal in domain of respective noise. The comparative remark is made at conclusion ${ }^{19}$.
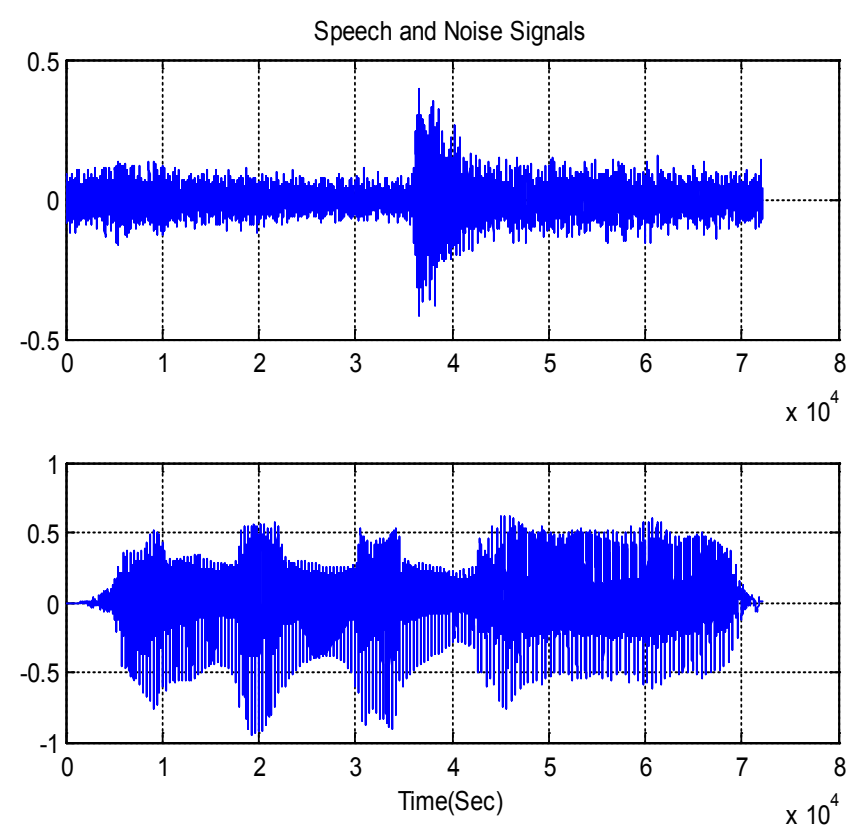

$4(a)$
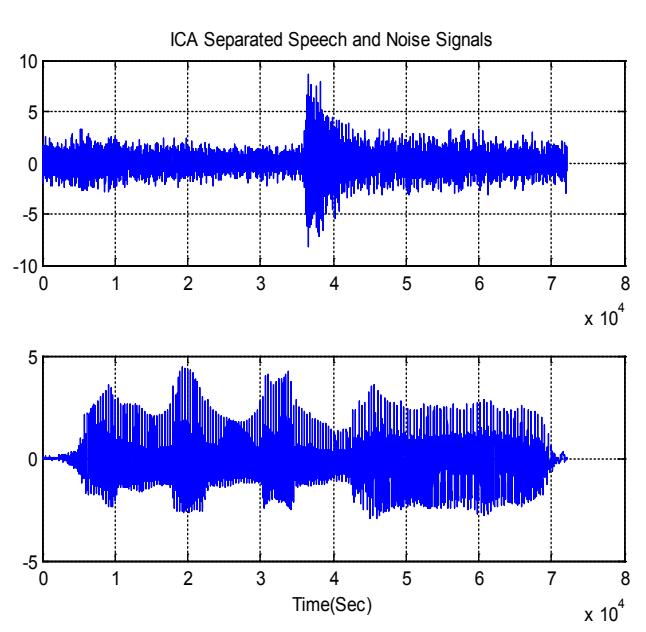

$4(\mathrm{~b})$


$4(c)$
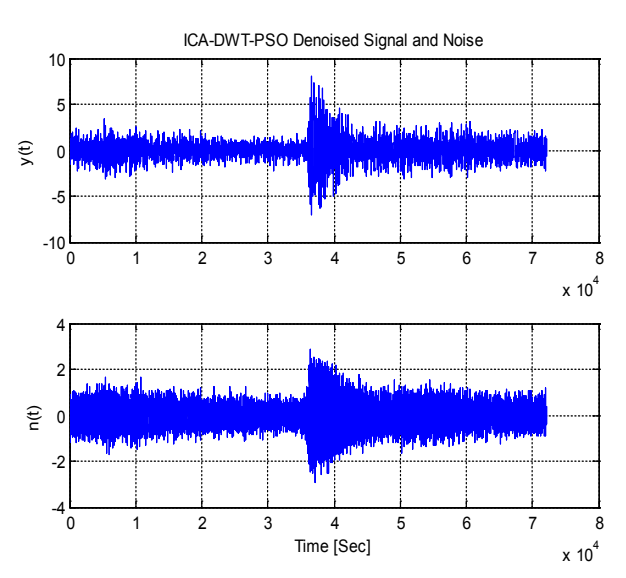

$4(d)$

Figure 4. The mixing and denoising of periodic noise and speech signal. (a) are the speech and noise signals that underwent mixing, (b) represents the ICA separated ICA and noise (c) filtering of signal using ICA and DWT (d) PSO based DWT to denoise signal. 
Table 1. Comparison of different algorithms for 'lalala' in 'diesel engine' domain

\begin{tabular}{|l|c|c|}
\hline Method & SNR $(\mathrm{dB})$ & SQI \\
\hline ICA & 15.879 & 0.99982 \\
\hline ICA-DWT & 16.177 & 0.99966 \\
\hline ICA-DWT-PSO & 20.632 & 0.99957 \\
\hline
\end{tabular}

Table 2. Comparison of different algorithms for 'hello girl' in 'diesel engine' domain

\begin{tabular}{|l|c|c|}
\hline Method & SNR $($ dB $)$ & SQI \\
\hline ICA & 13.009 & 0.99962 \\
\hline ICA-DWT & 13.362 & 0.99885 \\
\hline ICA-DWT-PSO & 15.959 & 0.99842 \\
\hline
\end{tabular}

Table 3. Comparison of different algorithms for 'welcome' in 'diesel engine' domain

\begin{tabular}{|l|c|c|}
\hline Method & SNR $(\mathrm{dB})$ & SQI \\
\hline ICA & 6.949 & 0.99993 \\
\hline ICA-DWT & 7.41 & 0.99975 \\
\hline ICA-DWT-PSO & 14.202 & 0.99965 \\
\hline
\end{tabular}

Table 1, Table 2 and Table 3 shows the comparative analysis of noise separation in industrial noise (diesel engine) with lalala ,hello girl and welcome speech samples respectively .Different method is implemented and compared with ICA,ICA-DWT and novel technique (i.e. ICA-DWT-PSO).The performance evaluation parameter SNR(DB) reflects the increase in gain with novel technique. Diesel engine mixed with welcome speech shows $7 \mathrm{db}$ gain in hybrid one $(\mathrm{ICA}+\mathrm{DWT}+\mathrm{PSO})$ compare to ICA. Diesel engine mixed with hello girl speech shows 3 dbgain in hybrid one (ICA+DWT+PSO) compare to ICA. Diesel engine mixed with lalala speech shows $5 \mathrm{db}$ gain in ICA compare to hybrid one. Comparing the SQI form table 1,2 and 3 , it shows slightly better than results in hybrid one (ICA+DWT+PSO) and ICA 20 .

Similarly the proposed algorithm is tested with different noise samples and their results are tabulated in Tables followed. The Table 4 provides results for test in Car noise, Table 5, Table 6 shows the performance in large industrial noise, Table 7 shows results of Robotic machinery noise sample, Table 8 , Table 9 signal denoising results in factory noise and Table 10 shows the performance $n$ machinery noise.
Table 4. Comparison of different algorithms for 'lalala' in 'car noise' domain

\begin{tabular}{|l|c|c|}
\hline Method & SNR $(\mathrm{dB})$ & SQI \\
\hline ICA & 1.5494 & 0.99658 \\
\hline ICA-DWT & 1.6643 & 0.98287 \\
\hline ICA-DWT-PSO & 5.4479 & 0.99251 \\
\hline
\end{tabular}

Table 5. Comparison of different algorithms for 'lalala' in 'large industrial noise' domain

\begin{tabular}{|l|c|c|}
\hline Method & SNR $(\mathrm{dB})$ & SQI \\
\hline ICA & 15.536 & 0.99997 \\
\hline ICA-DWT & 15.363 & 0.99991 \\
\hline ICA-DWT-PSO & $\mathbf{1 8 . 8 9 6}$ & $\mathbf{0 . 9 9 9 2 7}$ \\
\hline
\end{tabular}

Table 6. Comparison of different algorithms for 'welcome' in 'large industrial noise' domain

\begin{tabular}{|l|c|c|}
\hline Method & SNR $(\mathrm{dB})$ & SQI \\
\hline ICA & 16.567 & 0.99998 \\
\hline ICA-DWT & 17.055 & 0.9999 \\
\hline ICA-DWT-PSO & $\mathbf{1 8 . 8 7}$ & $\mathbf{0 . 9 9 9 9 2}$ \\
\hline
\end{tabular}

Table 7. Comparison of different algorithms for 'lalala' in 'robotic machinery noise' domain

\begin{tabular}{|l|c|c|}
\hline Method & SNR $(\mathrm{dB})$ & SQI \\
\hline ICA & 0.79774 & 0.99599 \\
\hline ICA-DWT & 2.6087 & 0.99439 \\
\hline ICA-DWT-PSO & $\mathbf{1 1 . 2 4 7}$ & $\mathbf{0 . 9 9 7 7 1}$ \\
\hline
\end{tabular}

Table 8. Comparison of different algorithms for 'hello girl' in 'factory noise' domain

\begin{tabular}{|l|c|c|}
\hline Method & SNR $(\mathrm{dB})$ & SQI \\
\hline ICA & 15.947 & 0.99994 \\
\hline ICA-DWT & 16.022 & 0.9998 \\
\hline ICA-DWT-PSO & $\mathbf{1 9 . 1 4 4}$ & $\mathbf{0 . 9 9 9 3 9}$ \\
\hline
\end{tabular}

Table 9. Comparison of different algorithms for 'welcome' in 'factory noise' domain

\begin{tabular}{|l|c|c|}
\hline Method & SNR $(\mathrm{dB})$ & SQI \\
\hline ICA & 6.797 & 0.9995 \\
\hline ICA-DWT & 6.9273 & 0.99876 \\
\hline ICA-DWT-PSO & $\mathbf{8 . 1 5 0 4}$ & $\mathbf{0 . 9 9 8 4 2}$ \\
\hline
\end{tabular}


Table 10. Comparison of different algorithms for 'lala' in 'machinery noise' domain

\begin{tabular}{|l|c|c|}
\hline Method & SNR $(\mathrm{dB})$ & SQI \\
\hline ICA & 7.7541 & 0.99979 \\
\hline ICA-DWT & 8.6987 & 0.99976 \\
\hline ICA-DWT-PSO & $\mathbf{1 6 . 6 2 5}$ & $\mathbf{0 . 9 9 9 7 1}$ \\
\hline
\end{tabular}

For the non-periodic noise, the mixing of signals is performed at 5 levels of sound to noise ratio. 3 such signals are acquired (column 1 of Table 11) and their intial SNR are recorded (column 2). These signals after filtering with ICA and ICA-DWT and ICA-DWT-PSO, output SNR of each is signal is compared. Table 11 indicates that if ICA-DWT-PSO is selected over ICA and ICA-DWT for speech signal filtering, the output SNR will be higher than both these algorithms for at least in every aspect $\frac{19}{}$.

Table 11. Comparison table of SNR for 3 sample speech signals and 6 input noise SNR values of non periodic signals

\begin{tabular}{|c|c|c|c|c|}
\hline \multirow{2}{*}{$\begin{array}{l}\text { Input } \\
\text { signal }\end{array}$} & \multirow{2}{*}{$\begin{array}{c}\text { Input } \\
\text { SNR } \\
\text { (db) }\end{array}$} & \multicolumn{3}{|c|}{ Algorithms Output SNR (dB) } \\
\hline & & ICA & $\begin{array}{l}\text { ICA- } \\
\text { DWT }\end{array}$ & $\begin{array}{l}\text { ICA- } \\
\text { DWT } \\
\text {-PSO }\end{array}$ \\
\hline \multirow{5}{*}{$\begin{array}{l}\text { Speech } \\
\text { signal } 1\end{array}$} & $3 \mathrm{db}$ & 6.4006 & 6.8967 & 8.5724 \\
\hline & $8 \mathrm{db}$ & 6.4006 & 6.8967 & 8.5724 \\
\hline & $13 \mathrm{db}$ & 6.4877 & 6.8012 & 7.6413 \\
\hline & $0 \mathrm{db}$ & 5.4806 & 6.2812 & 8.056 \\
\hline & $-4 \mathrm{db}$ & 5.1267 & 5.9748 & 7.2076 \\
\hline \multirow{5}{*}{$\begin{array}{l}\text { Speech } \\
\text { signal } 2\end{array}$} & $3 d b$ & 12.595 & 13.099 & 13.13 \\
\hline & $8 \mathrm{db}$ & 11.905 & 12.557 & 15.598 \\
\hline & $13 \mathrm{db}$ & 11.292 & 12.035 & 15.584 \\
\hline & $0 \mathrm{db}$ & 11.605 & 12.232 & 15.341 \\
\hline & $-4 d b$ & 12.028 & 12.545 & 15.633 \\
\hline \multirow{5}{*}{$\begin{array}{l}\text { Speech } \\
\text { signal } 3\end{array}$} & $3 \mathrm{db}$ & 12.19 & 13.907 & 13.124 \\
\hline & $8 \mathrm{db}$ & 14.012 & 15.653 & 20.063 \\
\hline & $13 \mathrm{db}$ & 18.065 & 19.072 & 21.192 \\
\hline & $0 \mathrm{db}$ & 9.1211 & 11.184 & 18.338 \\
\hline & $-4 \mathrm{db}$ & 14.246 & 15.718 & 20.685 \\
\hline \multirow{5}{*}{$\begin{array}{l}\text { Speech } \\
\text { signal } 4\end{array}$} & $3 \mathrm{db}$ & 6.1701 & 6.8657 & 7.0184 \\
\hline & $8 \mathrm{db}$ & 5.5212 & 6.1035 & 7.2901 \\
\hline & $13 \mathrm{db}$ & 6.6057 & 6.8283 & 6.8283 \\
\hline & $0 \mathrm{db}$ & 6.4259 & 6.849 & 6.849 \\
\hline & $-4 d b$ & 6.2896 & 6.8255 & 6.8255 \\
\hline
\end{tabular}

\section{Conclusion}

This paper is a complete research on its part, considering both the periodic and aperiodic forms of noise for speech signal denoisng in industrial environment. Industrial noise is considered here due to its intensity and hazards on human beings. The paper evident the requirement of ICA to separate periodic noise from speech signals. To enhance the performance of system ICA separated signal is processed with DWT and found that SNR of signal improves but correct level of decomposition and perfect threshold value for shrinking is needed and that was found using PSO algorithm. System tested in 5 noise cases (4 periodic and one aperiodic with different SNRs) depict the superiority of proposed algorithm over conventional ones. The 'lalala' and 'welcome' speech signals have highest SNR and in rest of cases the SNR of proposed system is always greater than or equal to $100 \%$ from existing methods in domain of periodic noise. The rough averaging of results for periodic noise indicates that average SNR for ICA is $10 \mathrm{~dB}, \mathrm{ICA}+\mathrm{DWT}$ is $11 \mathrm{~dB}$ while for ICA+DWT+PSO is $15 \mathrm{~dB}$. In rest of cases and in periodic noise, the proposed technique has the highest SNR and Sound Quality Index. For the range of input signals and noise environment, the optimization of PSO to filter the speech signals has best SNR compared to existing methods.

\section{References}

1. Choi J, Yeom J, Chang A, Byun Y, Kim Y. Hybrid pansharpening algorithm for high spatial resolution satellite imagery to improve spatial quality. Geoscience and Remote Sensing Letters. 2013; 10(3):490-4.

2. Roman N, Woodruff J. Speech intelligibility in reverberation with ideal binary masking: Effects of early reflections and SNR threshold. The Journal of the Acoustical Society of America. 2013; 133(3):1707-17.

3. Zhou T. SNR. In Signal-to-Noise Ratio, Werner Dubitzky et al., editors. Springer New York; 2013. p. 1939-40.

4. Ljung R, Israelsson K, Hygge S. Speech intelligibility and recall of spoken material heard at different SNRs and the role played by working memory capacity. In Applied Cognitive Psychology. 2013; 27(2):198-203.

5. Balcan DC, Rosca J. For speech enhancement with missing TF content. Lecture Notes in Computer Science, Springer Berlin Heidelberg. 2006; 3889:552-60.

6. Hong L, Rosca J, Balan R. based single channel speech enhancement using wiener filter, Institute of Electrical and Electronics Engineers (IEEE) Symposium on Signal 
Processing and Information Technology (ISSPIT); 2003. p. 522-5.

7. Balakrishnalavu, Potnuru VA. Speech enhancement using constrained-ICA with Bessel features [Degree of Master of Science in Electrical Engineering thesis]. Sweden, Blekinge Institute of Technology; 2011 Jan.

8. Rutkowski T, Cichocki A, Barros AK. Speech enhancement using adaptive filters and approach. In the Proceedings of International Conference on Artificial Intelligence in Science And Technology (AISAT); 2000. p. 191-6.

9. Hong L, Rosca J, Balan R. Bayesian single channel speech enhancement exploiting sparseness in the ICA domain, In the 12th European Signal Processing Conference; 2004. p. 1713-16.

10. Mihov SG, Ivanov RM, Popov AN. Denoising speech signals by wavelet transform. Annual Journal of Electronics. 2009:712-5.

11. Chavan MS, Chavan MMN, Gaikwad MS. Studies on implementation of wavelet for denoising speech signal. International Journal of Computer Applications. 2010; 3(2):1-7.

12. Tomic M, Sersic D. Point-wise adaptive wavelet transform for signal denoising. Informatica. 2013; 24(4):637-56.

13. Sardy S, Tseng P, Bruce A. Robust wavelet denoising. Institute of Electrical and Electronics Engineers (IEEE) Transactions on signal processing. 2009; 49(6):1146-52.

14. Attias H, Deng L, Acero A, Platt JC. A new method for speech denoising and robust speech recognition using probabilistic models for clean speech and for noise. Proceeding Eurospeech. 2001 Sep; 3:1903-6.

15. Langlois D, Chartier S, Gosselin D. An introduction to independent component analysis: InfoMax and FastICA algorithms. Tutorials in Quantitative Methods for Psychology. 2010; 6(1):31-8.

16. Bijalwan A, Goyal A, Sethi N. Wavelet transform based image denoise using threshold approaches. International Journal of Engineering and Advanced Technology. 2012; 1(5):218-21.

17. Kennedy J, Eberhart RC. A discrete binary version of the particle swarm algorithm. In proceedings of Institute of Electrical and Electronics Engineers (IEEE) International Conference on Computational Cybernetics and Simulation. 1997 Oct; 5:4104-8.

18. Lakshmikanth S, Natraj KR, Rekha KR. A hybrid approach for speech signal denoising using ICA-DWT. International Journal of Electronics Communication and Computer Engineering. 2014; 5(6):1395-9.

19. Lakshmikanth S, Natraj KR, Rekha KR. Novel algorithm for noise cancellation in speech using ICA-EMD using PSO. International Journal of Signal Processing, Image Processing and Pattern Recognition. 2014; 7(6):345-58.

20. Lakshmikanth S. Novel approach for noise cancellation in industrial environment [Ph.D. thesis]. Department of Electronics Engineering, Jain University, Karnataka, India; 2015. 